\title{
Einflüsse von aktiver und rezeptiver Musiktherapie auf Kognition, Verhalten, Schlaf und allgemeine Befindlichkeit von Demenzpatienten - eine Pilotstudie*
}

\author{
Michaela Presch ${ }^{a} \quad$ Lukas Hart $^{\mathrm{b}} \quad$ Gerhard Tucek $^{\mathrm{b}} \quad$ Bernd Minnich $^{\mathrm{a}}$ \\ Werner Kullich ${ }^{c}$ Günther Bernatzky ${ }^{a}$ \\ aArbeitsgruppe für «Neurodynamics und Neurosignaling», Fachbereich für Organismische Biologie, \\ Naturwissenschaftliche Fakultät, Universität Salzburg, \\ bIMC Fachhochschule Krems GesmbH, Krems, \\ 'Ludwig Boltzmann Cluster für Rheumatologie, Balneologie und Rehabilitation, Saalfelden, Österreich
}

\section{Schlüsselwörter}

Demenz $\cdot$ Musiktherapie $\cdot$ Kognition $\cdot$ Verhalten $\cdot$ Herzratenvariabilität, HRV · Schlafqualität · Patientengerechtes Musikabspielgerät

\section{Zusammenfassung}

Hintergrund: In dieser Pilotstudie wurde die Wirkung von aktiver und rezeptiver Musiktherapie auf Senioren mit Demenz $(n=3$; Mini-Mental State Examination (MMSE): 15/16/20 von 30) untersucht. Probanden und Methoden: Beide Interventionsformen sollten primär für sich genommen, in weiterer Folge aber auch auf etwaige Gemeinsamkeiten und Unterschiede hin untersucht werden. Mögliche Effekte der Musiktherapie auf Kognition und Verhalten der Probanden waren von Interesse. Ausserdem wurden zusätzlich innovative und in diesem Bereich bisher kaum erprobte Messinstrumente eingesetzt: Geräte zur Messung der Herzratenvariabilität (HRV) sowie Schlafuhren (Actiwatches) zur Erhebung der Schlafqualität. Dies geschah auch im Hinblick auf eine mögliche Implementierung dieser Methoden in weiteren, grösser angelegten Studien. Im Zuge der rezeptiven Musiktherapie wurde ein (auf ältere Menschen adaptierter) Prototyp eines Musikabspielgeräts verwendet, dessen Tauglichkeit für Menschen mit Demenz getestet und optimiert werden sollte. Die Probanden durchliefen 1 Woche Kontrollmessungen (Baseline) ohne Musiktherapie, 2 Wochen aktive Musiktherapie, 1 Kontrollwoche ohne Intervention, 2 Wochen rezeptive Musiktherapie und 1 abschliessende Kontrollwoche. Während des gesamten Zeitraums (insgesamt 7 Wochen) wurde die Schlafqualität der Teilnehmer erhoben und zusätzlich an je 3 Tagen pro Woche die HRV gemessen. Zu bestimmten Zeitpunkten wurden mithilfe von Fragebögen (MMSE, Neuropsychiatrisches Inventar (NPI)) der Demenz-

*Gewidmet Herrn Prof. Dr. Hans Adam (Universität Salzburg) zum 85. Geburtstag. schweregrad bzw. etwaige Befindlichkeits- und Verhaltensauffälligkeiten ermittelt. Ergebnisse: Der MMSE-Wert verbesserte sich bei allen 3 Probanden, am stärksten nach der aktiven Musiktherapie. Das NPI ergab tendenziell Verbesserungen während bzw. nach der aktiven Musiktherapie, wobei sich keine durchgängigen Tendenzen während der rezeptiven Musiktherapie zeigten. Bezüglich der HRV führte die aktive Musiktherapie zu einer Aktivierung und Vitalisierung (erhöhte HRV), während aus den Messergebnissen der rezeptiven Musiktherapie kein durchgängiger Effekt hervorgeht. Die Schlafqualität wurde in den Wochen der rezeptiven Musiktherapie negativ und während der aktiven Musiktherapie und der Kontrollphasen positiv beeinflusst.

\section{Keywords}

Dementia · Music therapy · Cognition · Behaviour - Heart rate variability, HRV · Sleep quality · Patient-friendly music player

\section{Summary}

Effects of Active and Receptive Music Therapy on Cognition, Behaviour, Sleep, and General Mood of Dementia Patients - a Pilot Study

Background: In this pilot study, the influence of active and receptive music therapy on elderly probands with dementia $(n=3$; Mini-Mental State Examination (MMSE): 15/16/20 of 30) was examined. Subjects and Methods: Both forms of therapy should be evaluated for their effectiveness as well as for similarities and differences. Possible effects of music therapy on cognition and behaviour were of interest. Also, instruments for measuring heart rate variability (HRV) and sleeping clocks (Actiwatch) for measuring quality of sleep were used and tested for their subsequent use in major studies. In the course of receptive music therapy, a prototype of a music player (adapted to the needs of elderly people) was used, which has already been improved and is

Univ--Prof. Dr. Günther Bernatzky

Arbeitsgruppe für «Neurodynamics und Neurosignaling» Fachbereich für Organismische Biologie

Naturwissenschaftliche Fakultät, Universität Salzburg

Hellbrunnerstrasse 34, 5020 Salzburg, Österreich

Tel. +43 662 8044-627, Fax -616, guenther.bernatzky@sbg.ac.at 
currently being used in an ongoing study. Each participant underwent 1 week of control measurements (baseline) without music therapy, 2 weeks of active music therapy, 1 week without intervention (control), 2 weeks of receptive music therapy, and a final control week. During the whole period ( 7 weeks), sleep quality data were collected, and additionally, HRV was measured 3 times per week. At certain points, degrees of dementia as well as changes of mood and behaviour were determined by using questionnaires (MMSE and Neuropsychiatric Inventory (NPI)). Results: The MMSE value improved in all 3 subjects, with the highest results after active music therapy. The NPI resulted by trend in improvements during and after active music therapy; no consistent trends were shown during receptive music therapy. Regarding HRV, active music therapy led to activation and vitalization (increased HRV), whereas receptive music therapy showed no consistent effect. The quality of sleep was negatively affected during the weeks of receptive music therapy, yet increased during active music therapy and the control phases.

\section{Mots-clés}

Démence - Musicothérapie - Cognition - Comportement .

Variabilité du rythme cardiaque, VRC · Qualité du sommeil . Lecteur de musique adapté au patient

\section{Résumé}

Influences de la musicothérapie active et réceptive sur la cognition, le comportement, le sommeil et l'état psychologique général de patients déments: une étude pilote

Objectif: Dans cette étude pilote, l'effet de la musicothérapie active et réceptive sur des personnes âgées atteintes de démence $(n=3$; Mini-Mental State Examination (MMSE): 15/16/20 sur 30) a été analysé. Participants et Méthodes: Les deux formes d'intervention devraient en premier lieu être considérées de manière individuelle; mais, par la suite, leurs éventuels points communs et différences devraient aussi être analysés. Les possibles effets de la musicothérapie sur la cognition et le comportement des sujets ont été notables. De plus, des instruments de mesure innovateurs et, jusqu'à présent, à peine testés dans ce domaine ont été utilisés en complément: des appareils de mesure de la variabilité du rythme cardiaque (VRC), de même que des moniteurs de sommeil (Actiwatches) pour enregistrer la qualité du sommeil. Ces méthodes ont aussi été appliquées en vue de leur possible implémentation dans de futures études de plus grande envergure. Au cours de la musicothérapie réceptive, un prototype (adapté aux personnes plus âgées) d'un lecteur de musique dont l'aptitude devrait être testée et optimisée pour les personnes atteintes de démence a été utilisé. Les sujets ont suivi 1 semaine de mesures de contrôle (référence) sans musicothérapie, 2 semaines de musicothérapie active, 1 semaine contrôle sans intervention, 2 semaines de musicothérapie réceptive et 1 semaine contrôle finale. Pendant toute la période $(7$ semaines au total), la qualité du sommeil des participants a été enregistrée et la VRC a été mesurée en plus 3 jours par semaine. Le degré de sévérité de la démence ou d'éventuelles particularités psychologiques et comportementales ont été déterminés à certains moments à l'aide de questionnaires (MMSE, inventaire neuropsychiatrique (NPI)). Résultats: Chacun des 3 sujets a présenté une amélioration du score du MMSE, la musicothérapie active ayant été suivie de l'amélioration la plus forte. Le NPI a révélé une tendance à l'amélioration pendant ou après la musicothérapie active alors qu'aucune tendance générale n'est apparue pendant la musicothérapie réceptive. En ce qui concerne la VRC, la musicothérapie active a entraîné une activation et une revitalisation (augmentation de la VRC) alors qu'aucun effet général n'est ressorti des mesures de musicothérapie réceptive. La qualité du sommeil a été influencée de manière négative pendant les semaines de musicothérapie réceptive et de manière positive pendant la musicothérapie active et les périodes de contrôle.

\section{Einleitung}

Im Verlauf einer Demenzerkrankung kommt es zu einer Schädigung und zum Verlust von Nervenzellen bzw. ihren Verbindungen, und das Gehirn beginnt, eine geringere Leistung aufzuweisen. Neue Eindrücke und Informationen können von den Betroffenen nur mehr eingeschränkt gespeichert und abgerufen werden, und auch die Erinnerung an ältere Gedächtnisinhalte verblasst zunehmend. Optisch-räumliche Störungen, Sprachstörungen, motorische Störungen sowie Schwierigkeiten im Erkennen von Personen und Gegenständen nehmen zu, für Erinnerungen existiert keine zeitliche Ordnung mehr und eine hochgradige Vergesslichkeit tritt ein [1]. Im Zusammenhang damit kommt es häufig zu affektiven und sozialen Veränderungen der Persönlichkeit sowie zu einer Überforderung bei der Lösung von diversen Alltagsproblemen. Demgegenüber scheint jedoch gerade das «musikalische Gedächtnis» bzw. der Sinn für die «Sprache der Musik» auch im fortgeschrittenen Krankheitsstadium noch weitgehend erhalten zu bleiben [2].

Die bekannteste und häufigste Form ist die AlzheimerDemenz. Bei Alzheimer-Patienten weist das Gehirngewebe deutlich weniger Nervenzellen und Synapsen auf als ein gesundes Gehirn. Die klinische Manifestation der Alzheimer-Demenz beginnt typischerweise im höheren Lebensalter. Neuropathologische Veränderungen wie Amyloidplaques und Ablagerungen von Tau-Protein sowie neurofibrilläre Tangles sind bekannt und liefern aufschlussreiche Informationen zur Pathophysiologie der Erkrankung. Rund 25\% der senilen Demenz vom Alzheimertyp werden autosomal dominant vererbt [3].

Musik spricht den Menschen auf so vielfältige Art und Weise an wie kaum ein anderes Phänomen. Aufmerksamkeit, (Selbst-)Wahrnehmung, Befindlichkeit, Kognition, Gedächtnis und Phantasie, aber auch Motorik und diverse physiologische Abläufe werden in der aktiven wie rezep- 
tiven Auseinandersetzung mit Musik gefordert bzw. gefördert. Als therapeutisches Hilfsmittel kann Musik ganz unterschiedlichen Zielsetzungen dienen. Bei Demenzpatienten wird Musik unter anderem eingesetzt, um die Orientierung zu unterstützen, das Sozialverhalten zu fördern, das Sprachverständnis und die Sprachproduktion $\mathrm{zu}$ verbessern, Ängste abzubauen, das Wohlbefinden zu verbessern und um die Gedächtnisfunktionen zu unterstützen [4-7]. Whitcomb [8] empfiehlt den Einsatz von Musik zum Abbau von Erregung, Verwirrung, Spannung, Ruhelosigkeit, Depression, Einsamkeit, Angst, Isolation und negativem Selbstbild. Musik kann sowohl aktivierend und ermutigend als auch beruhigend und tröstend wirken - nicht nur auf die Patienten, sondern in weiterer Folge auch auf Angehörige und Pflegepersonal [9]. Bekannte Musik aktiviert - auch noch bei Menschen mit demenziellen Gedächtnisstörungen - eine Vielzahl von (oft sehr positiv besetzten) biografischen Assoziationen und Erinnerungen. Das «musikalische Gedächtnis» ist über viele verschiedene Gehirnareale verteilt und somit sehr beständig; es kann die Degeneration einzelner neuronaler Funktionen teilweise überdauern bzw. kompensieren [2]. Das Sprachvermögen verschlechtert sich bei Menschen mit Demenz zunehmend, die musikalischen Fähigkeiten bleiben jedoch weitgehend erhalten [10].

Ziel dieser Pilotstudie war es, in Form von einzelnen Falluntersuchungen Vorarbeit zu leisten für weitere, grösser angelegte Studien in diesem Bereich. Die dabei gewonnenen Eergebnisse bestätigen bzw. falsifizieren für sich allein noch keine allgemeinen Hypothesen über den Effekt von Musiktherapie auf Senioren mit Demenz, vermitteln aber sehr wohl erste aufschlussreiche Tendenzen. Es wurde angenommen, dass der MMSE(Mini-Mental-State-Examination)-Kognitionswert nach den Musiktherapiebehandlungen höher (und damit besser) ist als davor, während negative Verhaltensauffälligkeiten reduziert sowie positive Auswirkungen auf das vegetative Nervensystem (manifestiert in der Herztätigkeit) und die Schlafqualität (längerer und erholsamerer Schlaf) erzielt werden sollten.

\section{Probanden und Methode}

Probanden

An der Studie nahmen 3 Personen teil (zwei Frauen und ein Mann mit leichter bis mittelschwerer Demenz (MMSE 15/16/20 von 30); durchschnittliches Alter 84 Jahre), die im Seniorenheim «Farmach» in Saalfelden (Salz burg/Österreich) wohnen. Bei allen Probanden erfolgten im Zeitraum der Studie keinerlei Umstellungen oder Änderungen der gewohnten Umgebung und der Pharmakotherapie. Die Ausschlusskriterien bei der Teil nehmerrekrutierung waren Schwerhörigkeit, Dehydratation, Angehörige fremder (Musik-)Kulturen, Abneigung gegenüber Musik, Berufsmusiker und schwere psychische Störungen/Krankheiten.

\section{Instrumente zur Datenerhebung}

\section{Fragebögen}

Die MMSE [11] wurde verwendet, um den Demenzschweregrad und eine eventuelle (und erwartete) Verbesserung der Kognition zu erheben. Der Test wurde zu Beginn der ersten Woche sowie am Ende der dritten und sechsten Woche (unmittelbar nach der jeweils letzten aktiven bzw. rezep tiven Musiktherapie) durchgeführt. Ein Wert unterhalb von 23 (von insgesamt 30) Punkten weist auf eine krankheitswertige Beeinträchtigung hin; bei 23-16 Punkten wird eine leichte Demenz, bei 16-7 eine mittel schwere Demenz und bei einem Wert unter 7 eine schwere Demenz diagnostiziert. Die MMSE ist das am häufigsten angewandte Screening-Ver fahren für Gedächtnisstörungen und wird unter anderem an der Spezialambulanz für Gedächtnisstörungen am Allgemeinen Krankenhaus der Stadt Wien (AKH) zur Demenzerkennung zusammen mit dem Uhren test eingesetzt.

Mithilfe des Neuropsychiatrischen Inventars (NPI) [12] sollten Auffälligkeiten in Verhalten und Befindlichkeit der Probanden im Verlauf der Studiendauer ermittelt werden. Der Fragebogen basiert auf Fremdbeurteilung und wurde vom jeweils zuständigen Pflegepersonal ausgefüllt. Der Gesamtwert setzt sich aus 12 Bereichen zusammen (Wahnvorstellungen, Halluzinationen, Erregung/Aggression, Depression, Angst, Euphorie, Apathie, Enthemmung, Reizbarkeit, abweichendes motorisches Verhalten, Schlaf- und Verhaltensstörungen, Appetit). Eine Zunahme des Gesamtwerts bedeutet eine Verschlechterung, eine Abnahme eine Verbesserung des Zustands der getesteten Person. Das NPI wurde am Ende jeder Woche, d.h. insgesamt 7-mal, erhoben.

\section{Messgeräte}

Die Herzratenvariabilität (HRV) wurde mithilfe eines speziellen Messgeräts [13] ermittelt, um die vegetativen Reaktionen (z.B. Entspannung Stress usw.) bzw. die psychische und physische Vitalität der Probanden während der Musiktherapiesitzungen möglichst objektiv (in Ergänzung zum NPI-Fragebogen) zu untersuchen. Das Herz eines gesunden Menschen reagiert auf diverse Einflüsse mit fein abgestimmten Veränderungen («Variationen») der Herzschlagfolge - diese sogenannte HRV stellt also ein zuverlässiges Mass für die allgemeine Anpassungsfähigkeit und Flexibilität eines Organismus dar [13]. Mithilfe der HRV-Messungen ist es unter anderem möglich, Aussagen über das biologische Alter, das Leistungspotenzial, die Regenerationsfähigkeit, das Aktivitätsniveau und die vorhandenen Ressourcen eines Menschen zu treffen. Das verwendete Messgerät ist ein modernes und mobiles EKG-Gerät (Medilog AR12 Digital Holter Recorder; Schiller Medilog, Belmont, Australien), das durch ein Kabel mit 3 Elektro den, die am Oberkörper der Probanden angebracht werden, verbunden ist.

Um die Auswirkung der Musiktherapie auf die Schlafqualität der Patienten zu erheben, wurden «Actiwatches» (Schlafuhren; CamNtech Ltd. Cambridge, Grossbritannien) [14] benutzt. Mithilfe dieser Geräte ist es möglich, Aussagen über die Schlafdauer bzw. Schlafqualität zu treffen. Die "Actiwatch» ist ein kompaktes und leichtes Messgerät (getragen wie eine Armbanduhr) zur Erhebung von physischen Bewegungen. Sie wird über jeweils $72 \mathrm{~h}$ auf der nicht dominanten Hand getragen und sammelt Bewegungsdaten, die gespeichert und dann mithilfe eines Lesegeräts in ein Computerprogramm [14] übertragen werden.

\section{Musikabspielgerät}

Für die rezeptive Musiktherapie wurde ein Musikabspielgerät der Firma Reditune (Salzburg, Österreich) [15] eingesetzt. Es handelt sich dabei um einen Prototyp, der speziell an die Bedürfnisse älterer Menschen angepasst wurde. Dieses Gerät besitzt grössere Knöpfe, um die Bedienung übersicht licher und einfacher zu gestalten. Im Rahmen dieser Studie wurde das Gerät jedoch vom diensthabenden Personal und nicht von den Probanden selbst bedient, um zu gewährleisten, dass die angebotene Musik auch tat sächlich in dem dafür vorgesehenen Zeitraum gehört wurde. Das bestehen de Modell wurde zudem (Stand: April 2010-April 2011) in einer von der Österreichischen Forschungsgemeinschaft finanzierten Studie (Projekt nummer 823580) verbessert und noch stärker an die Bedürfnisse von Demenzpatienten angepasst, um in weiterer Folge eine selbstständige Bedienung zu ermöglichen. 
Da es sich um eine Pilotstudie mit entsprechend geringer Teilnehmerzahl handelte, wurde das statistische Design der Intrakontrolle gewählt; d.h. jeder Proband durchlief alle verschiedenen Versuchs- bzw. Kontrollphasen und konnte auf diese Weise mit sich selbst "verglichen» werden (wie reagiert ein und dieselbe Person auf aktive Musiktherapie, rezeptive Musiktherapie und interventionsfreie Zeiten).

Über den gesamten Studienzeitraum hinweg wurden die Schlafuhren getragen; die HRV-Messungen wurden an 3 Tagen pro Woche für jeweils ca. 3 h durchgeführt. Während der Versuchswochen fanden in dieser Zeit auch die Therapieeinheiten statt. Zu Beginn der Studie sowie am Ende der dritten und sechsten Woche (unmittelbar nach der jeweils letzten aktiven bzw. rezeptiven Musiktherapiesitzung) wurde der MMSE-Wert erhoben. Dazu wurden die Teilnehmer einzeln in ihren Zimmern befragt. Der NPIFragebogen wurde insgesamt 7-mal am Ende jeder Woche vom zuständigen Pflegepersonal ausgefüllt.

$\mathrm{Zu}$ Beginn durchliefen alle Probanden eine Kontrollwoche (Baseline) mit Messungen ohne Musikintervention. In den Wochen 2 und 3 erfolgte die aktive Musiktherapie, die von einem Musiktherapeuten (in Ausbildung) in den Einzelzimmern der Probanden durchgeführt wurde. Die Gestaltung der aktiven Musiktherapie orientierte sich an den individuellen Bedürfnis sen und Fähigkeiten der Teilnehmer, die jeweils ganz unterschiedliche Musik-Bezüge hatten. Sie sollten hier möglichst aktiv in ein gemeinsames zwischenmenschlich-musikalisches Geschehen miteinbezogen werden. Im Anschluss an die Phase der aktiven Musiktherapie gab es eine Kontrollwoche ohne Intervention; die Messungen wurden jedoch fortgesetzt Darauf folgte in den Wochen 5 und 6 die rezeptive Musiktherapie, wobei die Probanden (ebenfalls an 3 Tagen pro Woche) allein in ihrem Zimmer lagen oder sassen und über Köpfhörer eine speziell zusammengestellte CD («Musik für Senioren», Dauer ca. 30 min) hörten. Die verwendete Musik nimmt auf das Langzeitgedächtnis der Probanden Bezug. Es handelt sich dabei um bekannte Lieder aus deren Jugendzeit, die bei älteren Menschen (laut einer vorangegangenen Studie in einem Salzburger Seniorenheim) vorwiegend positiv besetzt sind. Die Musikabspielgeräte wurden vom diensthabenden Personal ein- und ausgeschaltet. Nach der rezeptiven Musiktherapie folgte in der darauffolgenden Woche eine abschliessende Kontrollwoche ohne Musikintervention.

\section{Statistische Analysen}

\section{$H R V$}

Die HRV-Messungen wurden mithilfe eines Web-basierten Analysesystems (Darwin Scientific Software der Firma TOM Medical) [16] ausgewertet und mittels Spektralanalyse bildhaft dargestellt.
Auf Basis des Computerprogramms SigmaStat (Systat, Chicago, IL, USA) [17] wurde eine Statistik über die einzelnen Schlafparameter erstellt. Die 3 Teilnehmer wurden über alle Tage einer Interventionsform (aktive Musiktherapie, rezeptive Musiktherapie und Kontrolle) gepoolt. Getestet wurde mittels Varianzanalysen (Kruskal-Wallis und ANOVA) und Posthoc-Testverfahren. Das Signifikanzniveau lag bei 95\% $(p<0,05)$. Die Auswertung erfolgte nur über die Schlafzeit.

\section{Ergebnisse und Diskussion}

Die MMSE-Werte verbesserten sich bei allen 3 Probanden um 1-5 Punkte (Abb. 1). Die grösste Verbesserung im Vergleich zur Vormessung zeigte sich nach der aktiven $\mathrm{Mu}$ siktherapie (2-5 Punkte); nach der rezeptiven Musiktherapie stiegen die Werte um 0-1 Punkte. Aufgrund der geringen Fallzahl von nur 3 Probanden können daraus, wie bereits erwähnt, keine generellen Aussagen abgeleitet werden.

Aus den NPI-Gesamtwerten (Tab. 1) von Proband 1 und Proband 2 geht eine deutlich positive Entwicklung (Verringerung des Gesamtwerts) während der aktiven Musiktherapie (Woche 2 und 3) hervor; der «Ausreisser» in der zweiten Messung von Proband 3 bezieht sich lediglich auf einzelne Unmutsäusserungen des Probanden bezüglich des Anlegens und Tragens der Messgeräte. Für den Zeitraum der rezeptiven Musiktherapie (Woche 5 und 6) ergibt sich ein vergleichsweise heterogenes Bild ohne durchgängige Tendenzen.

Bei genauerer Betrachtung der einzelnen Parameter zeigen sich kontinuierliche positive Entwicklungen etwa im Bereich Depression, Apathie/Gleichgültigkeit, Schlaf/ nächtliche Verhaltensstörungen sowie Wahnvorstellungen [18]. Ähnlich wie bei den MMSE-Werten gab es auch hier die grössten Verbesserungen relativ zur Vormessung während der Wochen der aktiven Musiktherapie.

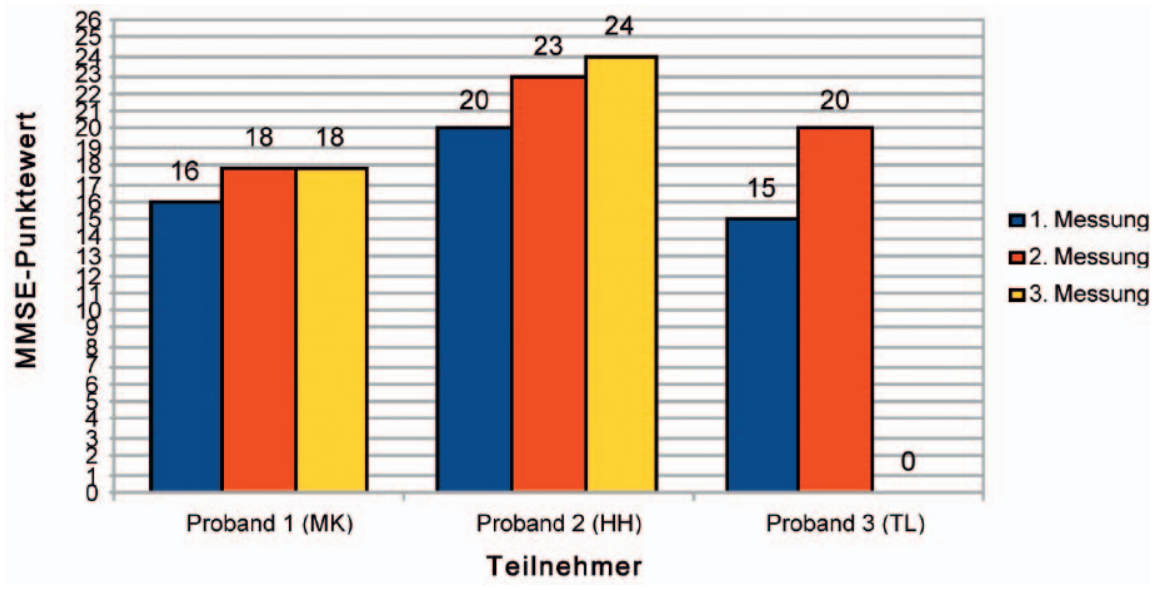

Abb. 1. MMSE-Werte zu den 3 Messpunkten. Proband 3 wurde gegen Ende der Studie in ein Krankenhaus verlegt, weshalb die dritte Messung nicht mehr durchgeführt werden konnte. 
Tab. 1. NPI-Gesamtwerte an den 7 Messpunkten $(\mathrm{KH}=$ Verlegung in ein Krankenhaus)

\begin{tabular}{lrrrrrrr}
\hline NPI & Messung & & & & & & \\
\cline { 2 - 7 } & 1 & 2 & \multicolumn{1}{c}{3} & 4 & \multicolumn{1}{c}{5} & \multicolumn{1}{c}{6} & 7 \\
\hline P1 (MK) & \multicolumn{1}{c}{30} & 14 & 0 & 0 & 12 & 0 & 9 \\
P2 (HH) & 18 & 19 & 11 & 6 & 0 & 33 & 6 \\
P3 (TL) & 0 & 6 & 0 & 0 & 0 & KH & KH \\
\hline
\end{tabular}

Bezüglich HRV führte die aktive Musiktherapie zu einer Aktivierung und Vitalisierung (erhöhte HRV) der Probanden, während aus den Messergebnissen der rezeptiven Musiktherapie kein durchgängiger Effekt hervorgeht. Die Anwendung der HRV-Geräte hatte zum Teil auch negative Auswirkungen auf die Teilnehmer. Es kam gelegentlich zu Unmutsäusserungen und Beschwerden über die «Verkabelung» und das Mit-sich-Herumtragen der Messgeräte. Das wiederholte Aufkleben der Elektroden führte teilweise zu leichten Hautirritationen auf der Applikationsstelle.

Die aktive Musiktherapie hatte deutlich positive Auswirkungen auf das Schlafverhalten - die Schlafeffizienz sowie die Gesamt-Aktivitätswerte waren während dieser 2 Wochen am besten. Auch die Kontrollphasen ergaben relativ gute Schlafwerte; hingegen kam es im Zeitraum der rezeptiven Musiktherapie zu einer Verschlechterung der Schlafqualität. Das Tragen der Schlafuhren bereitete den Teilnehmern zu Beginn mitunter auch Schwierigkeiten und erforderte eine gewisse Zeit der Gewöhnung. Letztendlich erwies sich das Gerät jedoch als leicht handhabbar und somit auch geeignet für den Einsatz im Bereich der Geriatrie bzw. Altenpflege.

Eine detaillierte Darstellung der 3 Falluntersuchungen und der Ergebnisse dieser Studie liefert Hartl [18]. Grundsätzlich gilt es dabei immer auch die vielfältigen äusseren Einflüsse unabhängig von der therapeutischen Intervention mit zu berücksichtigen, die in keiner derartigen Untersuchung gänzlich eliminiert werden können. Negative wie positive Erlebnisse mit dem Pflegepersonal, der Familie oder anderen Mitgliedern der Gemeinschaft, klimatische Einflüsse und Ähnliches können die Ergebnisse dieser Studie mit beeinflusst haben.

\section{Schlussfolgerung}

Im Rahmen dieser Studie wurden innovative Evaluierungsmethoden wie HRV und «Actiwatch» in den Bereich der geriatrischen Behandlung eingeführt, was sich als durchaus vielversprechend erwiesen hat und weitere Aufmerksamkeit verdient.

Insgesamt ergeben die gewonnenen Daten ein relativ kohärentes Bild. Das Setting der aktiven Musiktherapie hat der Situation und den Bedürfnissen der Teilnehmer offenbar besser entsprochen als jenes der rezeptiven Musiktherapie. Das zeigt sich sowohl an den Ergebnissen der HRV- und der Schlafqualitätsmessungen als auch an den relativen Verbesserungen der NPI- und MMSE-Werte. Damit wird die Hypothese bekräftigt, dass die Wirkung von aktiver Musiktherapie nicht allein auf den Einfluss der jeweiligen Musik als solcher zurückgeführt werden kann. Vielmehr spielen dabei eine Reihe von zusätzlichen Komponenten eine massgebliche Rolle, allen voran die zwischenmenschliche Beziehung zwischen Patient und Therapeut. Für bestimmte therapeutische Zielsetzungen (wie z.B. Entspannung, Schmerzlinderung) kann hingegen auch ein rein funktionaler Einsatz von Musik ohne den Rahmen eines persönlichen Vertrauensverhältnisses sinnvoll und angebracht sein. Derartige musikmedizinische Ansätze gilt es jedoch sowohl in Hinblick auf ihre Vorgehens- und Wirkungsweise wie auch bezüglich ihrer Indikation von Musiktherapie im engeren Sinn zu unterscheiden, die jedenfalls mehr ist (bzw. sein sollte) als blosse «Verabreichung» von Musik [19].

Es konnte gezeigt werden, dass vor allem aktive Musiktherapie die Kognition, die Befindlichkeit und Vitalität sowie die Schlafqualität von Menschen mit Demenz weitgehend ohne unerwünschte Nebenwirkungen verbessern kann. Der potenzielle Beitrag der Musiktherapie zu einer Verbesserung der Lebensqualität von Menschen mit Demenz ist sowohl hinsichtlich ihrer Beforschung als auch ihrer institutionellen Verankerung bei weitem noch nicht ausgeschöpft.

Um darüber hinaus auch effektivere rezeptive Musikangebote für den geriatrischen Bereich zu schaffen, sollten zukünftige Forschungen stärker auf die individuellen musikalischen Vorlieben jedes einzelnen Probanden eingehen und darüber hinaus möglichst benutzerfreundliche Musikabspielgeräte einsetzen. Ein solches Gerät wird derzeit (Stand: November 2010) im Rahmen einer gross angelegten Studie entwickelt - basierend auf den Ergebnissen der vorliegenden Pilotstudie.

\section{Dank}

Wir danken der Stiftung Sokrates in der Schweiz (Präsident Armin Kohler) für deren finanzielle Unterstützung. Unser Dank gilt auch dem Pflegepersonal des Seniorenheims Farmach für seine tatkräftige Unterstützung und natürlich den Probanden selbst für ihre Teilnahme und Kooperation.

Ebenso danken wir der Forschungsförderungsgesellschaft (FFG) Österreich für die finanzielle Unterstützung der Studie «Primosente» (Projektnummer 823580) zur Weiterentwicklung des Musikabspielgeräts.

\section{Disclosure Statement}

Die Autoren bestätigen, dass keinerlei Interessenkonflikte im Zusammenhang mit diesem Artikel bestehen. 


\section{Literatur}

1 Hörmann B, Weinbauer B: Musizieren mit dementen Menschen. Ratgeber für Angehörige und Pflegende. München, Ernst Reinhardt, 2006.

2 Sacks O: Der einarmige Pianist. Über $\mathrm{Mu}$ sik und das Gehirn. Reinbek, Rowohlt, 2008, pp 376-377.

3 Okello A, Koivunen J, Edison P, Archer HA, Turkheimer FE, Någren K, Bullock R, Walker Z, Kennedy A, Fox NC, Rossor MN, Rinne JO, Brooks DJ: Conversion of amyloid positive and negative $\mathrm{MCI}$ to $\mathrm{AD}$ over 3 years: an $11 \mathrm{C}$-PIB PET study. Neurology 2009;73:754-760.

4 Aldridge D: Music and Alzheimer's Disease - assessment and therapy. Discussion paper. J R Soc Med 1993;86:993-995.

5 Aldridge D: Alzheimer's disease: rhythm, timing and music as therapy. Biomed Pharmacother 1994;48:275-281.

6 Aldridge D: Music therapy and the treatment of Alzheimer's disease. Clin Gerontol 1995;16:41-57.
7 Aldridge D: Music therapy and the treatment of Alzheimer's disease. J Clin Geropsychol 1998;4:17-30.

8 Whitcomb J: «I would weave a song for you»: therapeutic music and milieu for dementia residents. Activities Adaptation Aging 1994;18:57-74.

9 Pomeroy V: Immobility and severe dementia: when is physiotherapy appropriate? Clin Rehabil 1994;8:226-232.

10 Swartz K, Walton J, Crummer G, Hantz E, Frisina R: Does the melody linger on? Music cognition in AD. Semin Neurol 1989;9:152158.

11 Folstein MF, Folstein SE, McHugh PR: «Minimental state». A practical method for grading the cognitive state of patients for the clinician. J Psychiatr Res 1975;12:189-198.

12 Cummings JL, Mega M, Gray K, RosenbergThompson S, Carusi DA, Gornbein J: The Neuropsychiatric Inventory: comprehensive assessment of psychopathology in dementia. Neurology 1994;44:2308-2314.
13 Autonom Health ${ }^{\circledR}$ GesundheitsbildungsGmbH. www.autonomhealth.com.

14 Cambridge Neurotechnology Ltd. www.camntech.com.

15 Reditune Audio \& Video. www.reditune.at

16 Medilog. www.tom-medical.com.

17 Systat. Statistical and Graphical Software. www.systat.com.

18 Hartl L: Musiktherapie in der Behandlung von Demenz - philosophische und psycho-physiologische Annäherungen. Diplomarbeit. Gföhl, Institut für Ethnomusiktherapie, 2010.

19 Hartl L: Musik im Kontext von Heilung, Medizin und Therapie. Historische und gegenwärtige Perspektiven; in Musalek $\mathrm{M}$, Poltrum M (Hrsg): Ars medica. $\mathrm{Zu}$ einer neuen Ästhetik in der Medizin. Berlin, Parodos, 2010. 